\title{
Euphemistic Expressions: A Challenge to L2 Learners
}

\author{
Meher.ul.nissa Zaid ${ }^{1}$, Fatima Batool ${ }^{2}$, Asia Khan ${ }^{3}$, Sumara Hina Mangla ${ }^{4}$ \\ Pakisthan
}

*Corresponding Author: Meher.ul.nissa Zaid, Pakisthan

\begin{abstract}
Although euphemism, being an effective communication device, gives the native speakers the opportunity to sound polite in the most impolite situations, their presence often generates confusion among the non-native speakers for whom the language has more to do with conveying the exact meaning than implying something beyond the obvious. The present study sets out to investigate whether euphemism asserts the intended meanings to L2 learners and to what extent they remain able to comprehend the meaning of a euphemistic expression. A sample of a hundred students at Bachelor level is chosen to investigate the underlying research questions. The data, on the understanding of euphemistic expressions regarding death, anger, urination, pregnancy and getting fired from job, is gathered through multiple methods, including observation, questionnaire and a group discussion. The study is also backed by empirical findings and factual details. The calculation of the frequencies of the correct answers to different euphemistic expressions reveals that the selected English euphemistic expressions are subject to complexity and vagueness for L2 learners. Even most of the correct answers were mere guesswork. The qualitative analysis of data reveals some very significant reasons working behind the vagueness of such expressions including educational background and medium of education. The elements of complexity and misunderstanding can be characterized by the intransparent nature of the words and to some extent interference of L1. The study speculates that the ever increasing proliferation of euphemisms by teachers as well as by classroom material affects student's learning and understanding of language when used in daily lives.
\end{abstract}

Keywords: Euphemism, Complexity, Vagueness, L2 Learners

\section{INTRODUCTION}

Language is a complex system of sounds that stands for words that code meanings. Communication via language takes place only, when the words generated by the sender convey the intended meaning to the receiver. Euphemism is the expression that shrouds the vulgarity or harshness of an experience with less offensive words. What stands for good speech among L1 learners often remains elusive in L2 learners, who are superficially acquainted with the language and have no knowledge of the culture that shaped that language. This self-exploratory study presumes that the semantic ambiguity of euphemism in a language could be a challenge for second language learners, characterized by its peculiar lexical forms. Though euphemisms have metaphorical use in language, their lexical forms represent the base form that eventually provides a bed rock upon which semantic values are carried out in second language learning context. English language has a wide range of euphemistic expressions developed in the course of years. Although Communication theorists and Linguists have been exploring their various dimensions, semantic ambiguity of euphemisms in second language acquisition appears to be a less attempted area. No study has been carried out on the students of English language at graduate level in Pakistan.

The term 'euphemism' is taken from Greek: the prefix 'eu' stands for 'good', 'well'; the stem 'pheme' for 'speak'; the suffix '-ism' for 'action or result'(www.saidwhat.co.uk(2003). 'Euphemism', speaking well or good speech, is an expression which substitutes a harsh or unpleasant word in order to lessen its intensity of harshness and to increase its agreeableness. The Oxford Companion Dictionary of English Language (1992) defines euphemism as an expression which replaces vulgar, blunt and offensive religious issues and makes them agreeable and less offensive. According to Oxford Dictionary, Euphemism is a roundabout $t$ word or expression replaced for the one taken to be too insensitive or blunt when that is referring to something unlikable or awkward. It allows the speaker to be polite and agreeable in the most disagreeable situations.

Samoskaite (2011) opines that the core objective of using euphemism in communication is to 
maintain a healthy social relationship in society. It substitutes the offensive and distasteful words with acceptable and polite expressions. To support this idea, four maxims of Grice (1975) can be taken into account. He suggests that a verbal exchange should be: first, „relevant“; second, as specific as the information is required, quantity maxim; third, "truthful" his quality maxim; and fourth unambiguous to avoid obscurity and ambiguity, maxim of manner. However, most of the time euphemistic expressions are found to be violating the maxims of quality and manner. Leech (1983) argues that politeness theory demands politeness, tactfulness and courtesy; therefore, verbal act should be performed in indirect manner to ensure least harshness and rudeness during the process of communication.

\subsection{Research Objectives}

This investigation has the following objectives:

- To explain the reasons of using euphemism in a language

- To identify the factors responsible for making euphemism deluding for L2 learners

\subsection{Research Questions}

The study seeks to investigate the following research questions:

Q.1. Why is Euphemism (expressions/phrases) challenging to the second language learners of English?

Q.2. Which are the factors working behind the elusiveness of Euphemisms?

\subsection{Significance of the study}

Because of the enhanced recognition of the factors that affect the acquisition of the second language learning by different linguists and scholars, many researches have been initiated to identify the factors that can be held responsible for the difficulties in the acquisition of second language, particularly English. However, the present study will be helpful to:

- the language teachers to look into their language teaching and learning methodologies;

- the curriculum designers to look for the new dimensions of euphemism and use them as one of the language tools;

- the ESL learners to go through their stock of knowledge of vocabulary and to concentrate upon their meanings of the words placed in particular psychological, social and cultural perspectives; and

- the language researchers to think over the concept of communicative competence acquired through euphemism.

A number of studies have been done on the different types and structures of euphemism. There has been extremely limited and insufficient knowledge about euphemistic awareness among second language (L2) learners. This study may prove a unique attempt in this regard.

Likewise, the study may also contribute to the research gap left by the previous researches and add some novice ideas in different dimensions. The understanding of students enrolled in Bachelors program is gauged, leaving behind room for more extensive study, aiming at the students of lower and higher level.

\section{LITERATURE REVIEW}

As far as the origin of euphemism is concerned, Samoskaite (2011) reports in her work that both Anglo Saxon society and renowned literary figures, such as, Chaucer, Shakespeare and George Blunt used euphemism in similar ways, but it got formally flourished in Middle English in early 17th century. With the passage of time it kept on refining its form and essence. Today it is evident that the whole world uses euphemism in its communication according to the historical, cultural and social background as well as geographic environment. Since Language is a changing phenomenon, changes are also expected in case of euphemism in terms of various demographic variables.

For Pavlenko (2006) euphemism protects speakers from unwanted emotional provocation. Brind \& Wilkinson (2008) highlight the unmentionable issues such as death, disability, bodily functions, war 
and sex as some of the domains euphemism deals with. Though it is noteworthy that some unmentionable taboos and offensive expressions do get acceptable in communication under the cover of euphemism, as projected by Jackall (2009) and Soles (2009), euphemism usually remains misleading and deceptive for speakers. Lacone (2003) seconds the idea by asserting that euphemism has covered and made overly complex the seemingly simple expressions. Further, Saphire (2008) names the birth of some euphemism confusing and controversial. The misleading euphemistic expression is, Trivers(2011) says, 'extraordinary rendition' that refers to 'torture along with kidnapping'. McArthur (2005) inscribes that frequent use of euphemism is very much part of newspapers. Chan, Chin and Nagami (2011) state that these newspapers are the source in ESL and EFL class rooms where readers find t euphemism as typical words are made unable to understand or to communicate the real gist of meaning.

To understand euphemisms one needs to understand the culture of that language with which euphemistic expressions are associated. Certain expressions are taken as taboos in all societies, substituting those expressions which are forbidden or must be avoided. Taboos are not universal, and some can have acceptance in many societies. Rawson (1995) asserts that euphemism is a culturallinguistic creation which exhibits its numerous mapping relations with culture. As a social tool, taboo helps to show courtesy, to fit to the proper context, and to articulate the ideas graciously (Allan \& Burridge,2007). Yon (2001) argues a vital feature of euphemism, whether it comes from the one's social false notion or from the social ethics. In the course of time, it loses its euphemistic character and presumes the full implication and connotation of the unique words that have been put out of place, becomes a taboo (unmentionable), and needs to be substituted by new euphemistic expression.

Nothing is permanent but change, argues Heraclitus, so does Palmer (1993), who believes that words change their meanings with the passage of time. It is the existence of taboos that regulates the formation of euphemisms. Certainly all languages have euphemisms that people use in avoiding being offensive. There are different areas where euphemism helps not only to disguise harshness, bitterness, and rudeness, but also to avoid unmentionable or covert social, religious and physical phenomenon such as death, sex, sexual organs, pregnancy, anger and getting hired and getting fired from job. Since euphemism repeatedly articulates something in an implied and covert way, sometimes this may cause vagueness and may leave behind double meanings. These concerns lead to confusion and bewilderedness among listeners.

Mencken (1974) maintains that euphemistic expressions are used in conversation because of three reasons: the desire to avoid giving pain, for example, when it is said that somebody has passed away or that somebody is gone instead of saying that someone is dead. Secondly, for the sake of prestige, for example, the use of the term "outsource" rather than firing an employee. Thirdly, for screening grudges and showing emotional control, for example when someone gets angry and says "foul or black mood" or just "go ballistic" instead of saying "go to hell" or "get lost". Pan (2013) believes that knowledge of euphemism helps in routine communication. Generally, euphemism of any language shares the cultural associations. L2 learners, however, contemplate into the links that words formulate among themselves in order to make a sense out of them. Since they do not belong to the same society they are expected to get confused.

\subsection{Some Prevalent Subjects of Euphemism}

Followings are some of the most commonly used areas/topics of Euphemism:

\subsubsection{Euphemism Related to Religion}

The oldest euphemism found is related to belief system. It was that words possessed great power. The ancient people were afraid that even talking about the evil spirits may attract them or result in bad luck. How this kind of euphemism came into existence is described by Keyes (29):

Being an extremely ferocious animal, the early northern Europeans regarded Bears as substitution for the idea of fear itself. Those people were afraid that expressing their genuine name may coax these fierce monsters. Rather, they discussed the nectar eater, the licker or granddad. The term "bear" advanced from an indirect term that implied the known one. Being recorded almost thousands of years back, it is the most seasoned doublespeak.

Owing to the fact that now there is no chance of coming across a bear except in zoos, the terror of bears has reduced. Therefore, the need to invent names to safely refer to bears no longer exists. In the 
same way words such as "hell" or "damn" are not taken as swear words because the idea that soul would go through hell after death is no more believed upon. "Jesus Christ", a term which was once considered an impiety (taken as a swear word), is not a taboo any more even among the believers. Nevertheless, the credulous fear that good fortune may be turned into bad luck if mentioned still persists in various forms.

\subsubsection{Euphemisms Related to Excretory Functions}

Since they are not euphemistic expressions, the terms for urination and defecation are used exclusively in medical sense. However the polite society prefers euphemism for referring to these basic needs. "Piss" and "shit" are the basic Anglo-Saxon words used for excretory functions; despite the fact that same word "piss" exists in the King James Bible (in Isaiah 36:12), they are considered vulgar and unacceptable as far as their general use is concerned.

The word manure, literally means "worked with the hands" (from the Latin: manus, manus - "hand"), refers to animal feces used as fertilizer for plants, also suggesting the mixing of manure with earth. The byproducts of elephants and other large herbivores are marketed by the zoos as Zoo Doo or Zoopoop. Interestingly, with the name Cock-a-Doodle Doo there is a brand of chicken manure. Also, "Baa Baa Doo" is a brand of sheep manure. Similarly, the word bull, or the abbreviation BS, often replaces the word bullshit in polite society. (The term bullshit is not taken in its literal sense "shit of a bull", but in a general sense it means lies or nonsense, thus, making it a dysphemism).To excuse oneself from the company, some lengthier paraphrases for ,excretion ${ }^{\text {ee }}$ are used, such as, to see a man about a dog (or horse), to powder one's nose, to drop the kids off at the pool or to release the chocolate hostages (regarded as dysphemism).

\subsubsection{Euphemism related to Death}

As stated by Keith and Burridge, death is a "fear based taboo". It is free of time, culture, religion and society. Around dead bodies, no one feels at ease or contented. Out of many reasons behind the uneasiness of the people, the fact that death has remained a mystery, something that can never be understood fully, is the most haunting. According to T. Pyles and J. Algeo, The origins and development of the English language ( $p p$ 216), just like the euphemistic terms used for dying, begetting and many excretory functions, the expressions used for diseases are embedded with the feelings of uneasiness and superstition. Events such as plague or sudden death, which the people remained failed to understand, were identified as God's punishment.

Such superstitious approach now a days does not exist; however, the anxiety related to death still continues. Death is also connected to the misery one feels at the death of a dear one, and a careful choice of words is required in order to lessen the pain or to avoid increasing it. Now a days, death mostly occurs in health care facilities, away from home, where it has become the duty of the doctors to disclose the heart breaking news to the kin of the deceased. The assumption, however, that doctors use euphemism only for the benefit of the patient or their family seems incorrect. Keyes (138) suggests that doctors themselves are more fearful of death as compared to other people; they go for the profession of medicine to overcome their own fear of death. In order to prevent the relatives of the deceased as well as him/herself from the trauma, they have to use implicit words to announce the death of the patient. Traditionally, doctors use expressions like, "she/he is gone", or "she/he is no more with us", or "she/he was lost by us "or "we could not save him/her".

On the contrary, media's frequent display of death may result in increased indifference to the sad news. Furthermore, different computer games in which the target is to kill as many enemies as possible may also lead to increased insensitivity. As already stated, most people in today's world die in absence of their closest relative, this correspondence in the choicest words make the very idea of death abstract in their minds. In a classical essay, Politics and the English Language (Horizon, 1946), Orwell (8) suggests when one thinks about a concrete object, one thinks wordlessly, and when one wants to describe something, that one has been visualizing, one may hunt about until one finds the exact words that appear to fit in. It means when one thinks about some abstract thing or Phenomenon, one is more likely to incline towards the use of words from the beginning-sometimes blurring or even altering the meanings altogether.

For doctors, there is no comparable, appropriate term/expression to reduce the impact of fatal diseases. Terms like "cluster of cells", abnormal growth", "mass", "troubling lab results" and "curious 
shadow on x-ray" are sometimes used instead of the word "cancer. Evidently, some euphemistic expressions are popular and widely used, while others remained failed to become a catch phrase.

\subsubsection{Euphemisms Related to Sex}

In almost every culture and age, just like death and illness, the concept of sex is also commonly and extensively euphemized. The reasons behind the use of these code words are entirely different from that of fear. Being a game of pleasure itself, different bodily cherished actions are expressed through various allusions. Resultantly, each society has a great variety of creative and pleasing euphemistic expressions.

The practice of euphemizing sexual activities is quite similar to the practice of children finding solutions to mysteries and riddles and talking in coded language, so that no one can intercept their conversation, and their secrets could remain intact. The use of such language confers upon the user a special kind of superiority along with a sense of satisfaction that one is capable enough to prove his/her intelligence.

The Greek expression $\alpha$ looíov (aidoion) and the Latin expression pudendum for the private parts truly signify "dishonorable thing". The larger parts of the body are alluded as crotches, groin and loins and are indirect when used to allude to the privates (to refer to the genitals). "Jerk off" is a word from Latin. Masturbate is derived from Latin, the word "manus" means hand and the word "sturbare" means to defile or debase. Generally, in the context of anal sex, the words "rosebud" and "starfish" are often used for anus in pornography. The widely-used set of polite euphemism for sex and relationship pattern in the U.S. is the baseball metaphors for sex. It includes terms like "hitting it off" for a good start to relationship, "running the bases" for progressing sexually in a relationship, and "striking out" for being unlucky with a love interest.

\subsubsection{Euphemism for Different Job Titles}

A few employments have confused titles that make them sound noteworthy than their regular names, making the use of code words a normal practice in job titles. Words, for example, architect can be incorporated into a large portion of these double speaks. Words such as engineer can be used for euphemism, though, in fact, the people who do the job are not accredited in engineering. Extreme cases, such as "transparent-wall maintenance officer" for window cleaner, or "sanitation engineer" for janitor are most often cited humorously. Another example is Henny oungma's joke that his brotherin-law, who claimed to be a diamond cutter used to mow the lawn at Yankees Stadium. There are more terms of respect used for the less extreme cases, such as custodian for janitor or administrative assistant for secretary. For a distasteful work, euphemism is brought in to describe the job, for example, "cemetery operative" for a gravedigger or "rodent officer" for a rat-catcher.

\section{Methodology}

This exploratory study falls in the middle of basic and applied research continuum. It is based on information rich paradigm. The primary aim is to investigate whether the use of euphemistic expression asserts the exact (intended) meanings to the L2 learners or if these euphemistic expressions remain elusive for L2 learners. The secondary aim is to identify the factors associated with deluding facts of euphemism for L2 learners. The population of this study is the Bachelors students enrolled in the winter 2015 semester for the major course of English Comprehension and Communication Skills at the University of Lahore.

Since the population was too large, the researcher selected 100 students falling between nineteen to twenty-one years of age, representing the total population of more than 300 students of BS. The selection was made through systematic random technique. Every third student had the chance to be a part of the sample. It was also convenient sampling as the researcher herself was a lecturer at the same institute and had an easy access to the data along with her familiarity with students" educational and cultural background. It is pertinent to mention that the participants were the true representatives of the population because of the observable fact that they all had a multilingual background and were learning English as their second language.

The data of this study is obtained through three instruments: Observation, a test and a group discussion.It has been indicated that the multiple-choice quiz/test is the most common and widely used assessment tool for the measurement of knowledge, ability and complex learning outcomes 
(Nicole, 2007,). Hence, the researcher choses this test to check the ability of the participants to recognize euphemistic expressions in English. In order to measure the understanding of the students, the researcher devised a structured quiz based on 25 multiple choice items. Further, a group discussion of the students in presence of the researcher was conducted in which participants spoke about the possible reasons for the ambiguity of the said expressions/terms. Taking into consideration the semantic ambiguity of euphemism, inference of shades of meanings could be an apparent hurdle for the L2 learners. Thus, in the following table, euphemism of five areas in English has been taken into account for this study.

Table1. Selected Euphemism according the specific domains

\begin{tabular}{|l|l|l|l|l|l|}
\hline & Death & Anger & Urination & Getting Fired From the Job & Pregnancy \\
\hline 1 & Pushing the daisies & Go ballistic & Take a leap & Outsource & In the pudding club \\
\hline 2 & Worm food & Blow a fuse & Spending a penny & Pink Slip & Up the duff \\
\hline 3 & Six feet under & Up in arms & Draining the tank & Surplus & In the family way \\
\hline 4 & Buy one way ticket & Foul Mood & To answer nature's call & Reduce & Pregger / Preggo \\
\hline 5 & Take a dirt nap & Black mood & Relieve oneself & Walk the plank & Bun in the oven \\
\hline
\end{tabular}

\subsection{Data Analysis}

After the data was collected, the frequency of the used euphemistic expressions by the respondents was calculated through the software called SPSS, and presented in tabulated form.

\section{DISCUSSION}

The researcher in order to analyze and interpret the data collected through questionnaires (written quizzes) calculated the frequencies of the correct answers (through SPSS), afterwards subjected these euphemistic expressions to further qualitative analysis through a group discussion of 25 randomly selected participants.

\subsection{Data Interpretation based on the Test (Quiz)}

After going through the data collection and analysis procedure, it is evident that L2 learners are not well versed in the use of euphemistic expressions, and only guessed what the expression might meant. The research shows that the only $26.4 \%$ of the total respondents were able to comprehend the euphemistic expressions related with the topic of death; $44.4 \%$ students could understand the expressions related to anger; only $22.8 \%$ comprehended the expressions related to pregnancy and just $21 \%$ understood euphemism used in firing from job whereas the concept of urination through Euphemism could be realized by $23.2 \%$ students only. Such a less number of L2 learners were able to understand the terms when used in the routine communicative practices.

Looking at the number of students who remained aloof from the concept wrapped in euphemism would bring to the surface that the majority of the respondents were unaware of the terms: $79 \%$ from getting fired from the job, $77.2 \%$ for pregnancy, 76.8 from urination, $73.6 \%$ from death and $55.6 \%$ from anger. It is also worth mentioning that overall $70 \%$ of the respondents felt elusive when these expressions were used but only in the case of anger the number of students was less than $60 \%$, which is still a large number, if we compare it with the other percentages.

In case of anger the expressions are mostly pointing to this end and comparatively understood by the students. That is why the respondents failed at judging the meaning and showed their elusiveness while attempting the test, whereas the least understanding was shown for the phrases used for firing from the job.

\subsection{Data Interpretation based on Observation (Group discussion)}

At first stage of methodology, in order to investigate the phenomenon (whether euphemism asserts the exact expressions or delude them for L2 learners), a group discussion was conducted in a selected group of the twenty five participants. The researcher herself was the mediator of the discussion. The qualitative data based on observation of the researcher elucidated that some of the selected euphemisms were quite transparent lexically, and L2 learners responded to them accurately, such as the euphemistic expressions for anger, go ballistic, blow a fuse, black mood, up in arms and in a foul mood represented the tone of anger to most of the participants, who were then able to quote right situations during the group discussion. 
Similarly, the euphemistic expressions for firing from the job (outsource, let go, pink slip, walk the plank and reduced) were adequately used and explained by six students using some suitable contexts, but the same subject remained vague for almost nineteen participants out of twenty five who found the expressions confusing. For this expression, a situation was given to the participants, and they were asked to interpret it. Twenty out of twenty five respondents were able to have a clear idea but again five got baffled.

Likewise, when the euphemistic expressions for urination was discussed in the group, four expressions (take a leap, draining the tank, spending a penny and relieve oneself) were not comprehended by twenty one learners; however, four participants understood but hesitated due to the connotation. They were the only ones who knew the real context.

On the contrary, a noteworthy demonstration was given on the understanding and use of euphemistic expressions on the subject of death by sixteen students. None of the participants was able to understand "worm food", "six feet under" and "sleeping with the fishes" and quoted odd examples and tried to translate their literal meanings instead of taking them as a unit, whereas "buy one way ticket" and "pushing up daisies" were easily understood by almost all of the participants.

While the last euphemistic expression for being pregnant was expressed through the terms in the pudding club, up the duff, the bun in the oven and pregger that were extremely difficult for almost all the participants. The researcher had to give the contextual explanation of the said terms, so that the expressions could become comprehendible for the language learners. While "in the family way" was the only expression that was grasped by all the respondents immediately.

While having a discussion with the students, the researcher came to know about different factors that were responsible for the incapability of the students in getting the meanings of the euphemistic expressions given in the quiz/test.

\section{KEY FINDINGS}

The key findings corresponding to the following research questions are mentioned below:

Q.1. Why is Euphemism (expressions/phrases) challenging to second language learners of English?

Q.2. Which are the factors working behind the elusiveness of Euphemism?

As it has been proved through the analysis of the test, more than half of the students were unable to comprehend the specific terms given to them. During the group discussion conducted among the respondents and the researcher, it was discovered that there were many factors working behind the (elusiveness) misunderstanding of these terms. The detail of some of these factors is given below:

\subsection{Difference in Social and Educational Background}

The most important factor for the poor understanding of the euphemism among L2 learners is the divide between the public and private sector educational institutes. Even the society itself is divided into classes. Being the representative of elite class, one has the opportunity to attend private schools like Aitcheson and Beacon House; one also has the fortune to study English Literature along with English Language at a very early stage of his/her learning. This somehow develops a familiarity of English Culture among these learners, thus, making them well versed in the use of euphemism.

Those who were unable to get the meanings of euphemistic terms, mostly belonged to the public sector schools and colleges. They had studied English as a compulsory subject, and most of the time studied it as another subject, taking it as a burden instead of a language. Most of them never had the opportunity to study English Literature, and were unaware of the English culture and could not grasp the meaning of the said terms.

\subsection{Lack of Exposure}

Students who studied English literature as a subject in the early stages of their language learning had first-hand knowledge and enough exposure to the customs, traditions and culture of foreign lands. This knowledge and exposure helped them learn and comprehend the second language better than those who were deprived of the same experience. 


\subsection{Different Shades of Meanings of Words in English}

Another reason that could be accounted for the misunderstanding of euphemism is inferences based on different shades of meanings of the words. All words are not used in the same way. They may not necessarily mean the same when said by someone else. Words have different shades of meanings with respect to each speaker and each experience. Second language learners, most of the time, run after the denotative meanings of the words, forgetting about their connotative explanations.

\subsubsection{In-Transparent Words}

Though Euphemism has metaphorical use in language, its lexical forms represent the base form that eventually provides a bed rock upon which semantic values are carried out in second language learning. These lexical forms become many times in-transparent, adding to the confusion and difficulty of the second language learners.

\subsection{L1 Interference/Mother Tongue Interference}

Mother tongue interference is one of the factors related to delusion of the euphemism as expressed in the quiz. It is a known fact that the resistance offered by the mother tongue hinders one's comprehension of second language.

\subsection{Difference in Perception and Communicative Competence}

No two people can think in the same way. Even twins display difference in the perception of the world around them though born to the same parents at the same time. Being the students of the same teacher, studying at the same institute and living under same conditions is no guarantee that both learners would show same performance. Their varying understanding of things and experiences of life would make students learn about the world in different ways. It is also one of the reasons that many participants could not do well in a quiz based upon Euphemistic terms.

\subsection{Untrained Teachers and Inappropriate Teaching Methods}

Another factor is the unimaginative and untrained teachers who remain unable to adequately teach a student. English Language in Pakistan is taught as a subject not as a language. Further, a great number of accidental teachers make the situation worse. Those who are either waiting for their results or have nothing better to do become teachers. In many cases teachers themselves are not clear about the concept of euphemism and cannot teach the same to their students. They do not have creative faculty to use the materials which could encourage and improve the understanding of the students on aforementioned subjects.

\subsection{Poor Curriculum and Faulty Teaching Materials}

The curriculum for language learning in our country is neither functional nor relying upon need analysis. Despite the fact that euphemism is among the building blocks of second language learning, it has not been added to second language learning materials. Curriculum needs to be context-based to enhance the comprehension of the learners regarding euphemism.

\section{CONCLUSION}

There is no denying the fact that use of euphemistic expressions has increased with time. Its effectiveness in toning down the harshness and pungent tone of the experiences as well as situations in order to maintain politeness is commendable. However, a technique which improves the way two native speakers communicate could be highly ambiguous, when used among non-native speakers, proving to be misleading for L2 learners. The use of Euphemism is a widespread and observable fact though linguistic in nature, but strongly woven into culture, religion, ideals and way of life. Euphemistic expressions are needed in communication sometimes to be polite and at others to be formal, hence, proving to be an essential part of a language. The frequent use of these expressions highlight the fluency and expertise of the competitive user.

The findings have asserted that when such (euphemistic) terms were used in front of the participants, most of them gave wrong answers showing complete inability to comprehend the meanings. The reason of this failing might be the non- inclusion of euphemism in the standard L2 teaching and learning process in Pakistan. The current study also revealed that by using cover-terms (euphemistic expressions) the intensity of feeling of loss and hurt can be reduced. 
Many researchers have studied euphemism from different perspectives, but for this study euphemism has been studied as a semantic phenomenon (vagueness) in SLA. The study suggests that in- transparent nature of euphemistic expressions could be a challenge for L2 learners, since the participants are nonnative speakers and are learning English as a second language. This semantic vagueness (value) of euphemisms could be a possible challenge as it is evident in some cases. However, there are certain limitations in the research. The study is conducted on a small scale so the result cannot be generalized. Besides, the use of euphemism is to be found in every human experience ranging from getting born to getting married, from starting learning to starting working, from growing up to growing old; there is vast range of euphemistic expressions that are used in English language and a more extensive study can be carried out to check their understanding by the second language learners to suggest measures that can be taken to improve the comprehension of English among Pakistani students.

\section{REFERENCES}

[1] Alkire, S. (2002). Introducing euphemisms to language learners. The Internet TESL Journal, 5. Retrieved February 7, 2014, from http://iteslj.org/.

[2] Allan, K., \& Burridge, K. (2007). Forbidden words. Cambridge: Cambridge University Press.

[3] Brind, J., \& Wilkinson, T. (2008). Funeral, Thanksgiving and Memorial Services. London: Hymns Ancient \& Modern Ltd.

[4] Chan, W., Chin, K., \& Nagami, M. (2011). Media in Foreign Language Teaching and Learning. Germany: Walter de Gruyter. http://dx.doi.org/10.1515/9781614510208

[5] Cieslicka, A. \& D. Singleton (2004). Metaphorical Competence and the L2 Learner. Writing and Vocabulary in Foreign Language Acquisition, 4, 69.

[6] Grice, H. P. (1975). Logic and conversation. In Cole, P. and J. Morgan (eds.) Syntax and semantics, 3. London: Academic Press

[7] Hojati, A. (2012). A Study of Euphemisms in the Context of English-speaking Media. International Journal of Linguistics, 4 .

[8] Jackall, R. (2009). Moral Mazes: The World of Corporate Managers. New York: Oxford University Press.

[9] Lacone, S. (2003). Write to the Point: How to Communicate in Business with Style and Purpose. The United States of America: Career Press.60.

[10] Leech, G. (1983). Principles of pragmatics. London: Longman.

[11] McArthur, T. (2005). Concise Oxford Companion to the English Language. New York: Oxford University Press.

[12] Mecncken, H. L.(1967). The American Language. New York.

[13] Nunberg, G. (2009). Years of Talking Dangerously. The United States of America: Public Affairs.

[14] Pan, Q. (2013). A Tentative Study on the Functions and Applications of English Euphemism. Theory and Practice in Language Studies,3.

[15] Pavlenko, A. (2006). Bilingual minds: emotional experience, expression and representation. Toronto: Multilingual Matters. 260.

[16] Rawson, H. (1995). Rawson's dictionary of euphemisms and other doubletalk. New York: Crown Publishers, Inc

[17] Samoskaite, L. (2011). 21st Century Political Euphemisms In English Newspapers: Semantic And Structural Study. Department of English Philology, Vilinus Pedagogical University. MA.

[18] Suzani, S. M. (2013). On the Iranian EFL Graduates' Recognition of Semantic Opaqueness of Euphemistic Expressions in English. International Journal of Language Learning and Applied Linguistics World,4 (2).

[19] Webster, N. (2000). Webster's Third New International Dictionary. New york.

\section{AUTHORS' BIOGRAPHY}

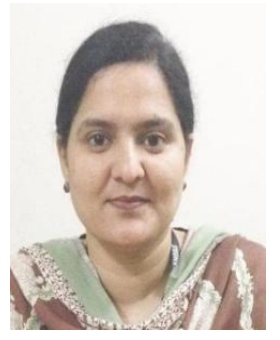

Meher ul nissa Zaid's teaching career at the University of Lahore spreads over ten years. She is currently teaching Literature and Language courses in the department of English. 


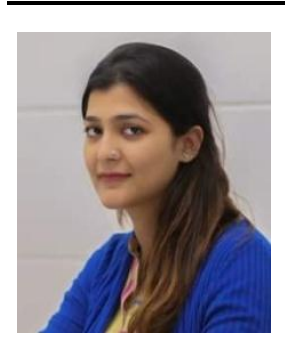

Fatima Batool is serving as Lecturer in the University of Lahore, Lahore. Besdies academic writing, she writes for leading newspapers and is currently working on her short-stories.

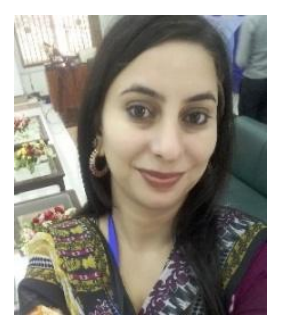

Sumara Hina is a Research scholar with over 5 years' experience of teaching \& Research in the department of English at the University of Lahore, Lahore.

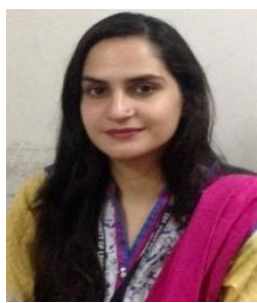

Miss Asiah Khan is working as lecturer in the department of English (Language and Literature) at the University of Lahore, Lahore.

Citation: Meher.ul.nissa Zaid, Fatima Batool, Asia Khan, Sumara Hina Mangla. "Euphemistic Expressions: A Challenge to L2 Learners "International Journal on Studies in English Language and Literature (IJSELL), vol 6, no.54, 2018, pp. 9-18. doi:http://dx.doi.org/10.20431/2347-3134.0605002.

Copyright: (C) 2018 Authors. This is an open-access article distributed under the terms of the Creative Commons Attribution License, which permits unrestricted use, distribution, and reproduction in any medium, provided the original author and source are credited. 